\title{
Usabilidad de las Páginas de Inicio de las Redes Sociales de Video
}

\author{
Usability of Homepages of the Social Video Networks \\ Cinthia Lima, Sandra Casas, Sebastián Alejandro Hernández \\ cinty.calderon.15@gmail.com, sicasas@uarg.unpa.edu.ar, sebastian.unpa@gmail.com \\ Instituto de Tecnología Aplicada (ITA), Universidad Nacional de la Patagonia Austral \\ Campus universitario Piloto Rivera s/n. Rio Gallegos, Santa Cruz
}

Recibido: 06/02/2020. Aceptado: 11/09/2020

\begin{abstract}
RESUMEN
En la última década los sitios web cuyo principal contenido son los videos, se han constituido como redes sociales de video (RSV). La popularidad de estas plataformas radica en el hecho de que millones de personas diariamente navegan por sus páginas, buscando, reproduciendo, subiendo, comentando y compartiendo videos propios y/o de otros usuarios. La usabilidad es un atributo de calidad del software y el nivel de usabilidad de los sitios web es uno de los factores clave a la hora de determinar el fracaso o éxito de un sitio web en el Internet. En este trabajo se presenta una evaluación de las páginas de inicio de las RSV Youtube, Vimeo, Dailymotion, Metacafe y Break con el objetivo de conocer los niveles de usabilidad de dichas páginas. Para llevar a cabo dicha evaluación se utilizó DESMET y para ello se confeccionó una lista de verificación de usabilidad para páginas de inicio con guías pertenecientes a diferentes aspectos de diseño. También se utilizaron herramientas de estadística básica. Este informe aporta tanto información cualitativa como cuantitativa, que identifica debilidades y fortalezas del diseño de las páginas de inicio de las RSV que están relacionadas con la usabilidad de las mismas.
\end{abstract}

Palabras Clave: usabilidad; página de inicio; web; redes sociales; video.

\begin{abstract}
In the last decade websites whose main content are videos, have become as social video networks (SVN). The popularity of these platforms lies in the fact that millions of people browse their pages daily, searching, playing, uploading, commenting and sharing their own or other users' videos. Usability is an attribute of software quality and the level of usability of websites is one of the key factors in determining the failure or success of a website on the Internet. This paper presents an evaluation of the home pages of the SVN Youtube, Vimeo, Dailymotion, Metacafe and Break in order to know the usability levels of these pages. To carry out this evaluation, DESMET was used, and an usability checklist for homepages was prepared with guides belonging to different design aspects. Basic statistical tools were used as well. This report provides qualitative and quantitative information, which identifies weaknesses and strengths of the design of the RSV home pages that are related to their usability.
\end{abstract}

Keywords: usability; homepage; web; video; social network. 


\section{INTRODUCCIÓN}

La calidad de software es fundamental en cualquier desarrollo de software y puede ser medida en diferentes niveles de acuerdo a su cumplimiento de los atributos de calidad descriptos en la norma ISO/IEC $25010^{1}$, la cual define que un producto de software de calidad debe tener las siguientes propiedades de calidad: funcionalidad, rendimiento, compatibilidad, compatibilidad, usabilidad, fiabilidad, seguridad, mantenibilidad y portabilidad.

El atributo de usabilidad o también conocido como facilidad de uso tiene que ver con el nivel de facilidad y amigabilidad que un software, en un contexto en específico, le puede ofrecer al usuario cuando este lo utiliza. Si el software no es lo suficientemente intuitivo o fácil de usar es posible que fracase, es decir que el usuario no lo quiera usar, aunque las funciones que ofrezca sean valiosas para él (Pressman, R. 2010). Es por esto que la usabilidad es clave para cualquier tipo de software, ya que de ello depende el éxito o fracaso del mismo.

Una de las aplicaciones de software más usadas en el mundo actualmente son las aplicaciones web o sitios web, los usuarios buscan en estos sitios una manera rápida y eficiente de realizar lo que desean (Nielsen, J. 2000). En las aplicaciones web, la usabilidad es un factor clave, debido a que éstas aplicaciones reciben por lo general usuarios de diferentes culturas y círculos sociales los cuales utilizan la aplicación para llegar a un mismo objetivo (Matos Mareño, R. 2013). En contraste, las aplicaciones de escritorio tienen un conjunto de usuarios definidos porque la aplicación realiza acciones específicas para ese tipo de usuarios. Por lo tanto, manejar la usabilidad en el ámbito de las aplicaciones web no es tan trivial como en cualquier aplicación de software.

Al momento de visitar un sitio web lo primero que se visita es la página de inicio del sitio, ésta es la cara del sitio y la primera impresión que el usuario tiene del sitio. Si la página de inicio no muestra de manera clara lo que ofrece el sitio y lo que los usuarios pueden hacer en él, éstos abandonan el sitio (Nielsen, J. 2012). La usabilidad en las páginas de inicio de los sitios web es muy importante, ya que es la página principal del sitio, si esta no es clara, fácil de usar y amigable para el usuario éste saldrá de la página directamente, y eso demuestra que el sitio no cumple con el atributo de calidad de la usabilidad.

En este artículo se trabajará en particular con los sitios web de video. Existen diversos sitios web de video, pero los que han tomado mayor relevancia recientemente son los sitios de redes sociales. Dentro de estos sitios se encuentra YouTube, Facebook, Twitter, entre otros. Estas redes sociales se caracterizan por que sus usuarios constantemente comparten todo tipo de contenidos multimedia ya sean fotos, videos, texto, audio, etc. Particularmente las Redes Sociales de Video (RSV) han adquirido una gran popularidad, la cual ha crecido exponencialmente en los últimos años. Estas redes sociales tienen como característica que el principal tipo de contenido multimedia que se comparte son videos. Algunas de ellas son el ya conocido YouTube, nombrado anteriormente, Vimeo y Dailymotion. En la mayoría de ellos los usuarios pueden subir videos y compartirlos con el resto de la comunidad. Las comunidades de estos sitios suelen ser muy grandes, recientemente un estudio de We are social $^{2}$ junto con Hootsuite mostró que Youtube tiene 1,900 millones de usuarios (octubre 2018), ocupando el segundo lugar en una escala junto con otras plataformas sociales. Esto

\footnotetext{
${ }^{1}$ ISO/IEC 25010:2011 - SquaRE - https://www.iso.org/obp/ui/\#iso:std:iso-iec:25010:ed-1:v1:en

${ }^{2}$ https://wearesocial.com/blog/2018/10/the-state-of-the-internet-in-q4-2018
} 
demuestra el nivel de popularidad y uso de este tipo de sitio web hasta la fecha. Por ende, la importancia de tener niveles altos de usabilidad en las páginas de inicio de estos sitios.

El objetivo de este trabajo consiste en analizar los sitios web de videos o RSV mediante inspecciones, con el fin de obtener información sobre los niveles de usabilidad de estos sitios, la metodología utilizada se encuadra en el método DESMET (Kitchenham, B. 1996). El trabajo se realiza sobre cinco RSV, realizando el análisis de la usabilidad de las páginas de inicio de estos sitios. Para ello se utiliza una lista de verificación de usabilidad aplicada a las RSV elegidas. La lista de verificación se define de acuerdo a las 113 guías de usabilidad de páginas de inicio de Nielsen, J. (2001). Además, con la información cuantitativa obtenida de la evaluación se realizaron diversos cálculos estadísticos básicos que facilitan la comparación y análisis general.

La estructura del artículo se organiza de la siguiente forma. En la Sección 2 se dará una definición general de usabilidad y conceptos relacionados. La Sección 3 presenta la metodología utilizada para el trabajo. En la Sección 4 se exponen los resultados obtenidos en el estudio. Finalmente, se presentan las conclusiones en la Sección 5.

\section{USABILIDAD}

La usabilidad es uno de los atributos de calidad a ser evaluados a la hora de definir el nivel de calidad de un producto de software. La usabilidad es el grado en el que el software es fácil de usar por un usuario, es decir, que tan entendible, aprendible y operable es (Pressman, R. 2010). La usabilidad no es un simple atributo de calidad de un sistema, también puede desglosarse en varios sub-atributos de usabilidad que pueden ayudar a entender mejor el concepto general de usabilidad. Los atributos de usabilidad son: facilidad de aprendizaje, está relacionado con el hecho de minimizar el tiempo que se requiere desde el no conocimiento de una aplicación hasta su uso productivo, de decir, que tan fácil es para los usuarios realizar tareas básicas la primera vez que se encuentran con el software; tiempo de respuesta, la capacidad del software de expresar los cambios de estado del usuario, es un factor muy variable, ya que depende de las características que tenga la computadora donde se encuentre el usuario; flexibilidad, tiene que ver con las formas que tiene el usuario de intercambiar la información con el sistema, la flexibilidad presente en el sistema implica brindar control al usuario, una capacidad de sustitución y capacidad de adaptación; robustez, sub-atributo vinculado con la necesidad de que el usuario cumpla con sus objetivos y que disponga del asesoramiento necesario; recuperabilidad, es el grado de facilidad con el que una aplicación le permite al usuario corregir una acción que dio algún error; sintetizabilidad, este factor tiene que ver con que el usuario sea capaz de captar cuando ocurra algún cambio de operación en el sistema; consistencia, es la capacidad de utilizar de la misma manera todos las funcionalidades del sistema, sea cualquiera el momento en el que se las necesite; y disminución de la carga cognitiva ${ }^{3}$, los aspectos cognitivos de la interacción proporcionan la necesidad que tienen los usuarios de confiar más en los reconocimientos que en los recuerdos (no tienen que recordar abreviaciones y códigos muy complicados), este último aspecto influirá en la disposición y el diseño de los distintos elementos de la interfaz gráfica del sistema (Perurena Cancio, L., Moráguez Bergues M. 2013).

\footnotetext{
${ }^{3}$ La cognición es la facultad de un ser vivo para procesar información a partir de la percepción, el conocimiento adquirido y características subjetivas que permiten valorar la información
} 
Este atributo de calidad, está asociado directamente con la interfaz gráfica de usuario (GUI, en inglés Graphical User Interface), ya que son éstas las que permiten a los usuarios utilizar las funcionalidades que les ofrece el software (Nielsen, J. 2012). La ausencia de este atributo de calidad en las GUIs puede llevar al fracaso del producto de software, debido a que, si el software no cumple con alguno de los factores mencionados anteriormente, es posible que el usuario no quiera usarlo. Una mayor usabilidad significa que sus procesos sean más eficientes, se reduce el tiempo y esfuerzos necesarios por parte del usuario para poder utilizar las funcionalidades que ofrece el software (Serrano Mascaraque, E. 2009).

Considerar a la usabilidad en el desarrollo del software como atributo de calidad es clave y tiene que estar presente para el éxito de un producto de software, también genera beneficios colaterales en el software. Un producto de software con un grado alto de usabilidad impacta directamente en la reducción de los costos de aprendizaje y por ende en los costos de asistencia y ayuda al usuario, también en los asociados al diseño, rediseño y mantenimiento. Otra ventaja, si se trata de aplicaciones web, está relacionada al aumento de la tasa de conversión de visitantes a clientes de un sitio web, por ejemplo. Al mismo tiempo puede mejorar la imagen y el prestigio de un sistema. También impacta en los usuarios, mejorando la calidad de vida, ya que reduce su estrés, incrementa la satisfacción y la productividad.

Todos estos beneficios implican una reducción y optimización general de los costos de producción, así como también un aumento en la productividad. La usabilidad presente en los sistemas de software permite mayor rapidez en la realización de tareas, menor riesgo o propensión a errores y reduce las pérdidas de tiempo. En general, lo que se mejora es la calidad del producto (Sanchez, W. 2011).

\subsection{Evaluación de la usabilidad}

Para hacer una evaluación de la usabilidad existen diversos métodos. En la actualidad no existe una unificación en cuanto a los métodos a utilizar para llevar a cabo la evaluación, pero si hay algunos mayormente nombrados y que se repiten entre autores. Los métodos se pueden clasificar en cuatros grandes categorías (Matos Mareño, R. 2013):

- Indagación: este tipo de evaluación permite identificar los requerimientos de usabilidad, los cuáles son indispensables en las etapas tempranas de un proceso de desarrollo. Ejemplos: Tormenta de ideas, Encuestas, etc.

- Inspección: en este tipo de métodos se realizan inspecciones críticas de una GUI en base a un conjunto de principios de diseño. Los principios de diseño pueden ser cualquier conjunto de reglas que describan propiedades de la interfaz. Ejemplos: Inspección de estándares, Seguimiento cognitivo, Guías y listas de verificación, Evaluación heurística, etc.

- Pruebas con usuarios: este método de evaluación hace partícipe al usuario para el uso de prototipos del sistema o se le proponen escenarios de uso. Ejemplos: Card sorting, Co-descubrimiento, etc.

- Automatizadas: este método hace uso de procedimientos computarizados para realizar la evaluación. Ejemplos: Validador de estructura, Análisis de logs y Eye and mouse tracking.

Los métodos pueden ser aplicados a todas las etapas de diseño y desarrollo, desde la definición del producto de software hasta cuando el producto ya está en funcionamiento. Al momento de elegir un método, se debe considerar el costo y las restricciones de tiempo (Sanchez, W. 2011). 


\subsection{Usabilidad web y páginas de inicio}

Los sitios web, compuestos por páginas web, son un tipo de software con características diferentes a los convencionales programas de escritorio. La característica que lo diferencia del software tradicional es que se desarrolla enfocándose en el usuario, en comparación con otros tipos de programas. Las páginas web están especialmente centradas en el diseño de una adecuada interfaz de usuario, ya que ofrecen principalmente información para el usuario. La presentación y la manera de acceder a la información que brinda la página es fundamental para poder cumplir con ese objetivo (Serrano Mascaraque, E. 2009). El concepto de usabilidad en los sitios web es clave ya que determina el éxito o fracaso del sitio, si el sitio no es fácil de utilizar los visitantes simplemente no navegarán por él y de lo contrario estos permanecerán.

Un sitio web "usable" es aquel que funciona de tal manera que un usuario con conocimientos medios puede utilizar el sitio para cumplir sus objetivos particulares que atenderán a sus necesidades, sin llegar a experimentar una sensación de frustración por tratar de usar el sitio (Matos Mareño, R. 2013). Para lograr obtener usabilidad en un sitio web se pueden distinguir algunos requisitos genéricos como la capacidad de comprensión del sitio global; los servicios de ayuda; capacidades estéticas y de interfaz, y servicios especiales (Pressman, R. 2010).

Según Krug, S. (2006) una de las primeras reglas a tener en cuenta a la hora de considerar la usabilidad de un sitio web es no hacer pensar al usuario. Esto significa que el usuario no debería hacerse interrogantes cuando se encuentra frente a alguna página del sitio web. Algunos ejemplos de los posibles interrogantes que se harían los usuarios son: ¿Por dónde empiezo?; ¿Por qué llamarán a esto así?; ¿Puede hacer clic en esto?; estos dos vínculos parecen ser lo mismo ¿lo serán realmente?, entre otros. Cuando el sitio no es lo suficientemente obvio, claro y fácil de entender hacen pensar a los usuarios y hacer esfuerzos por tratar de entender el sitio. La página de inicio del sitio web de una empresa u organización es su cara al mundo y la oportunidad para atraer y retener a cada visitante. Esta página es diferente a las demás páginas del sitio, una página de inicio bien construida proyectará una primera impresión general de lo que hay en el sitio a todo aquel que lo visite. Una página de inicio debe comunicar claramente el propósito del sitio y mostrar todas las opciones principales disponibles en el sitio web (Leavitt, M.O. y Shneiderman, B. 2004). El impacto que puede generar una página de inicio en el resultado final de una empresa u organización va más allá de las medidas de los ingresos del comercio electrónico, impacta también en el reconocimiento que puede llegar a obtener la organización u empresa dentro del mercado electrónico en el que está sumergido. La página de inicio de un sitio web es la más importante, ya que es el punto de partida para la mayoría de las visitas al sitio y por ende la más visitada (Nielsen, J. 2012). Y al ser la más visita, si la primera impresión que se tiene de la misma no resulta atractiva, usable y/o no sabe "venderse" al usuario que la está visitando, esto generará en el usuario el deseo instantáneo de buscar otro sitio web alternativo y debido a eso la organización u empresa perderá un cliente/visitante (Serrano Mascaraque, E. 2009).

\section{MATERIALES Y MÉTODOS}

En esta Sección se presentan los métodos y recursos empleados en el estudio. Se eligió la metodología de DESMET (Kitchenham, B. 1996) para la evaluación de los objetos de estudio, las RSV, del presente trabajo. La metodología DESMET está compuesta por un conjunto de métodos con sus respectivas herramientas que pueden ser aplicadas o utilizadas por una 
organización o grupo. DESMET propone en general tres tipos de evaluación: cuantitativas, cualitativas e híbridas. Además, el método define ciertos criterios técnicos que permiten determinar que método es el adecuado para la evaluación. Los criterios del método DESMET son: el contexto de la evaluación; la naturaleza del impacto esperado de la utilización del método o la herramienta; la naturaleza del objeto de evaluación, es decir, si es un método, una herramienta o un método genérico; el alcance del impacto del método o la herramienta; la madurez del método o herramienta; la curva de aprendizaje asociada al método o la herramienta; las habilidades de medición que posee la organización encargada de evaluar.

De acuerdo a los criterios citados se puede enmarcar la evaluación a realizar en este trabajo, dentro del tipo de evaluación híbrida (cuantitativa y cualitativa) y su vez del tipo caso de estudio cuantitativo, que significa la evaluación de un método/herramienta que fue usada o aplicada en un proyecto de software real, con el fin de también obtener información cualitativa al realizar la evaluación (Kitchenham, B. 1996). En este caso esos proyectos son los sitios web, particularmente las páginas de inicio de estos sitios, y el método o herramienta aplicada es una lista de verificación de guías de usabilidad. Para llevar a cabo el caso de estudio cuantitativo se hizo uso del método evaluación de usabilidad de inspección descrito en la sección anterior. Los resultados obtenidos fueron complementados con la aplicación de estadística básica.

Se realizó un conjunto de actividades para llevar a cabo la evaluación de las RSV. Primero se definieron cuales RSV iban a ser consideradas casos de estudio para el trabajo, en segundo lugar se confeccionó una lista de verificación de usabilidad de páginas de inicio, como tercera actividad se definió un sistema de medición porcentual para evaluar el cumplimiento de cada RSV con la lista de verificación de usabilidad. Y por último se estableció el uso de una fórmula estadística para calcular la desviación estándar de los resultados obtenidos para conseguir más información cuantitativa al respecto.

\subsection{Selección de casos de estudio (RSV)}

Primero se seleccionaron cinco RSV que se pueden apreciar en la Tabla 1. Se puede distinguir el nombre, año de creación de la RSV, país de origen y lo más importante la URL de la página de inicio de las RSV. Las RSV seleccionadas son las que posteriormente van a ser inspeccionadas para ver el nivel de cumplimiento de las mismas mediante una lista de verificación de usabilidad especificada más adelante. Particularmente se analizaron las páginas de inicio de las RSV sin tener un usuario registrado en la misma, para dicho análisis se consideró el estado de dichas páginas hasta abril de 2019.

Tabla 1: RSV seleccionadas

\begin{tabular}{|c|c|c|c|}
\hline Nombre & URL & Año de creación & País de origen \\
\hline Youtube & www.youtube.com & 2005 & Estados Unidos, California \\
\hline Vimeo & www.vimeo.com/watch & 2004 & $\begin{array}{c}\text { Estados Unidos, New York } \\
\text { City }\end{array}$ \\
\hline $\begin{array}{c}\text { Dailymotio } \\
\text { n }\end{array}$ & www.dailymotion.com & 2005 & Francia, París \\
\hline Metacafe & www.metacafe.com & 2002 & Estados Unidos, California \\
\hline Break & www.break.com & 1998 & Estados Unidos \\
\hline
\end{tabular}




\subsection{Confección de una lista de verificación de usabilidad}

Luego de seleccionar las RSV se procedió a elaborar una lista de verificación de usabilidad. La lista de verificación fue creada a partir de las 113 guías para páginas de inicio propuestas por Nielsen J. (2001), las cuales están categorizadas en los siguientes grupos: comunicación del propósito del sitio (7 guías); comunicación de la información de la empresa (9 guías); escritura del contenido (12 guías); revelación de contenido mediante ejemplos (4 guías); archivo y accesos a contenido del pasado (una guía); enlaces (6 guías); navegación (6 guías); búsqueda (6 guías); herramientas y atajos de tareas (3 guías); gráficos y animación ( 7 guías); diseño gráfico (6 guías); widgets de interfaz de usuario (3 guías); títulos de las ventanas (5 guías); URLs (4 guías); noticias y comunicados (4 guías); ventanas emergentes y páginas de ensayo (3 guías); publicidad (4 guías); bienvenidas (una guía); comunicación de problemas técnicas y manejo de emergencias (2 guías); créditos (2 guías); volver a cargar la página y actualizar (2 guías); personalización ( 2 guías); recopilación de datos del cliente (2 guías); fomento de la comunidad (2 guías); fechas y horarios (5 guías) ,y cotizaciones de acciones y números de visualización (4 guías).

Luego de analizar el significado y objetivo de cada guía, se descartaron 23 guías y se eligieron 90 para aplicar en este estudio. Los criterios para descartar 13 guías fueron dos, la guía no era aplicable a las características de las páginas de inicio de RSV, dado que especifica directrices para elementos no presentes en una RSV, y/o, para medir el nivel de complimiento de la guía se requiere acceso a información no disponible al público general o que era desconocida.

A continuación, se indican las Guías a aplicar al estudio y en el Anexo 1 las guías que fueron descartadas.

\section{Comunicación del propósito del sitio}

G01. Mostrar el nombre de la empresa y/o el logo en un tamaño razonable y en un lugar notable.

G02. Incluir una línea de etiqueta (eslogan) que resuma explícitamente que hace la empresa o el sitio.

G03. Enfatizar que hace que el sitio sea valioso desde el punto de vista del usuario, así como en qué se diferencia de los competidores clave.

G04. Enfatizar las tareas de prioridad más altas así los usuarios tienen un punto de inicio claro en la página de inicio.

G05. Designar claramente una página por sitio como la página de inicio oficial.

G06. En el sitio web principal de la empresa, no usar la palabra "sitio web" para referirse a nada que no sea la totalidad de la presencia web de la compañía.

G07. Diseñar la página de inicio para que sea claramente diferente a todas las demás páginas del sitio

\section{Comunicación de información sobre la empresa}

G08. Colocar información corporativa del grupo, como acerca de nosotros, relaciones con inversores, sala de prensa, empleo y otra información sobre la empresa, en un área distinta. 
G09. Incluir un enlace en la página de inicio a la sección "Acerca de nosotros" que brinde a los usuarios una descripción general de la compañía y enlaces a cualquier detalle relevante sobre productos, servicios, valores de la empresa, etc.

G10. Si desea obtener cobertura de prensa para su empresa, incluya el enlace "Sala de prensa" o "Sala de noticias" en la página de inicio.

G12. Incluir un enlace "Contáctenos" en la página de inicio que vaya a una página con toda la información de contacto de la empresa.

G13. Proporcionar un mecanismo de "retroalimentación", especificar el propósito del enlace como si será leído por el servicio al cliente o el webmaster, y así sucesivamente.

G14. No incluir información interna de la compañía (que está dirigida a los empleados y debe ir en el intranet) en la web pública.

G15. Si el sitio reúne información del cliente, incluir un enlace de "Política de privacidad" en la página de inicio.

G16. Explicar cómo gana dinero el sitio web si no es evidente.

\section{Escritura del contenido}

G17. Utilizar un lenguaje centrado en el cliente.

G18. Evitar el contenido redundante.

G19. No usar frases inteligentes ni jerga de marketing que haga que a las personas les cueste demasiado entender que se quiere transmitir.

G20. Usar mayúsculas consistentes y otros estándares de estilo.

G21. No etiquetar un área claramente definida de la página si el contenido es suficientemente autoexplicativo.

G22. Evitar las categorías y listas con viñetas de un solo elemento.

G23. Usar espacios sin descanso entre las palabras en frases que deben ir juntas para que sean escaneables y comprendidas.

G24. Solo usar lenguaje imperativo como "Ingrese una ciudad o código postal" para tareas obligatorias, o calificar la declaración apropiadamente.

G25. Deletrear las abreviaturas, los inicialismos y los acrónimos, y seguir inmediatamente por la abreviatura, en la primera instancia.

G26. Evitar los signos de exclamación.

G27. Utilizar todas las letras mayúsculas con moderación y no como un estilo de formato.

G28. Evitar usar espacios y puntuación inapropiadamente para enfatizar.

\section{Revelación de contenido mediante ejemplos}

G29. Usar ejemplos para revelar el contenido del sitio, en lugar de solo describirlo. 
G30. Para cada ejemplo, tener un enlace que vaya directamente a la página detallada de ese ejemplo, en lugar de a una página de categoría general de la que ese artículo es una parte.

G31. Proporcionar un enlace a la categoría más amplia junto al ejemplo específico.

G32. Asegurar que sea obvio que enlaces conducen a información de seguimiento sobre cada ejemplo y que enlaces conducen a información general sobre la categoría en su conjunto.

\section{Archivos y accesos a contenido del pasado}

G33. Facilitar el acceso a cualquier elemento que haya aparecido recientemente en la página de inicio, por ejemplo, en las últimas dos semanas o meses, proporcionando una lista de las características recientes, así como poner los elementos recientes en los archivos permanentes.

\section{Enlaces}

G34. Diferenciar enlaces y hacerlos escaneables. Comenzar los enlaces con la palabra que contiene información, porque los usuarios a menudo escanean la primera o dos palabras para compararlos.

G35. No utilizar instrucciones genéricas, como "Haga clic aquí" como nombre de enlace.

G36. No utilizar enlaces genéricos, como "Más ..." al final de una lista de elementos.

G37. Permitir que los colores del enlace muestren los estados visitados y no visitados.

G38. No usar la palabra "Enlaces" para indicar enlaces en la página.

G39. Si un enlace hace otra cosa que no sea ir a otra página web, vincular a un archivo PDF o lanzar un reproductor de audio o video, un mensaje de correo electrónico u otra aplicación, asegurar que el enlace indique explícitamente lo que sucederá.

\section{Navegación}

G40. Ubicar el área de navegación principal en un lugar altamente visible, preferiblemente directamente adyacente al cuerpo principal de la página.

G41. Agrupar los elementos en el área de navegación para que los elementos similares estén uno junto al otro.

G42. No proporcionar múltiples áreas de navegación para el mismo tipo de enlaces.

G43. No incluir un enlace activo a la página de inicio en la página de inicio.

G44. No utilizar palabras inventadas para las opciones de navegación de categorías.

G46. Usar los íconos en la navegación solo si ayudan a los usuarios a reconocer una clase de elementos inmediatamente.

\section{Búsqueda}

G47. Proporcionar a los usuarios un cuadro de entrada en la página de inicio para ingresar consultas de búsqueda, en lugar de solo darles un enlace a una página de búsqueda.

G48. Los cuadros de entrada deben ser lo suficientemente amplios para que los usuarios vean y editen consultas estándar en el sitio. 
G49. No etiquetar el área de búsqueda con un encabezado; en su lugar, poner el botón "Buscar" a la derecha del cuadro.

G50. A menos que las búsquedas avanzadas sean la norma en el sitio, proporcionar una búsqueda simple en la página de inicio, con un enlace a la búsqueda avanzada o consejos de búsqueda si los tiene.

G51. La búsqueda en la página de inicio debe buscar en todo el sitio de forma predeterminada.

G52. No ofrecer una función para "Buscar en la Web" desde la función de búsqueda del sitio.

\section{Herramientas y atajos de tareas}

G53. Ofrecer a los usuarios acceso directo a tareas de alta prioridad en la página de inicio.

G54. No incluir herramientas no relacionadas con las tareas por las que los usuarios visitan el sitio.

G55. No proporcionar herramientas que reproduzcan la funcionalidad del navegador.

\section{Gráficos y animación}

G56. Usar gráficos para mostrar contenido real, no solo para decorar la página de inicio.

G57. Etiquetar los gráficos y las fotos si su significado no está claro en el contexto de la historia que acompañan.

G58. Editar las fotos y los diagramas de manera adecuada para el tamaño de la pantalla.

G59. Evitar los gráficos con marcas de agua (imágenes de fondo con texto encima de ellos).

G60. No utilizar una animación con el único propósito de llamar la atención sobre un elemento en la página de inicio.

G61. Nunca anime elementos críticos de la página, como el logotipo, la línea de etiqueta o el título principal.

G62. Permitir que los usuarios elijan si desean ver una introducción animada del sitio.

\section{Diseño gráfico}

G63. Limitar los estilos de fuente y otros formatos de texto, como tamaños, colores, etc. en la página porque el texto sobre diseñado puede restar valor al significado de las palabras.

G64. Usar texto de alto contraste y colores de fondo para que el tipo del texto sea lo más legible posible.

G65. Evitar el desplazamiento horizontal a 1024x768.

G66. Los elementos de la página más críticos deben ser visibles "por encima de la tapa" (en la primera pantalla de contenido, sin desplazamiento) en el tamaño de ventana más prevalente. 
G67. Usar un diseño líquido (técnica de diseño web que permite adaptar el diseño de una página a la resolución horizontal y vertical que cada usuario ha configurado para su pantalla) ${ }^{4}$ para que el tamaño de la página de inicio se ajuste a diferentes resoluciones de pantalla.

G68. Utilizar logos con criterio.

\section{Widgets de interfaz de usuario}

G69. Nunca usar widgets para partes de la pantalla en las que no se quiere que la gente haga clic.

\section{Asegurar que los widgets sean cliqueables.}

G70. Evitar utilizar múltiples cuadros de entrada de texto en la página de inicio, especialmente en la parte superior de la página donde las personas tienden a buscar la función de búsqueda.

G71. Usar los menús desplegables con moderación, especialmente si los elementos en ellos no se explican por sí mismos.

\section{Títulos de las ventanas}

G72. Comenzar el título de la ventana con la palabra que contiene información, generalmente el nombre de la empresa.

G73. No incluir el nombre de dominio de nivel superior, como ".com" en el título de la ventana a menos que sea realmente parte del nombre de la empresa, como "Amazon.com".

G74. No incluir "página de inicio" en el título. Esto añade verborrea sin valor.

G75. Incluir una breve descripción del sitio en el título de la ventana.

G76. Limitar los títulos de las ventanas a no más de siete u ocho palabras y menos de 64 caracteres en total.

\section{URLs}

G77. Las páginas de inicio de sitios web comerciales deben tener la URL http://www.company.com (o una equivalente para su país o dominio de nivel superior no comercial).

G78. Para cualquier sitio web que tenga una identidad estrechamente relacionada con un país específico que no sea Estados Unidos, utilizar el dominio de nivel superior de ese país.

G79. Si se encuentra disponible, registrar nombres de dominio para deletreos, abreviaturas o errores ortográficos comunes.

G80. Si se tienen ortografías alternativas de nombres de dominio, elegir una como la versión autorizada y redirigir a los usuarios a ella desde todas las demás ortografías.

\section{Ventanas emergentes y páginas de ensayo}

G85. Llevar a los usuarios a la página de inicio "real" cuando escriban la URL principal o haga clic en un enlace al sitio.

\footnotetext{
${ }^{4} 4$ https://headways.com.mx/glosario-mercadotecnia/palabra/diseno-liquido/
} 
G86. Evitar ventanas emergentes.

G87. No utilizar las páginas de enrutamiento para que los usuarios elijan su ubicación geográfica a menos que se tengan versiones del sitio en muchos idiomas diferentes, sin un solo idioma dominante.

\section{Publicidad}

G91. Evitar utilizar convenciones de anuncios para mostrar características regulares del sitio.

\section{Bienvenidas}

G92. No dar, literalmente, la bienvenida a los usuarios al sitio.

\section{Créditos}

G95. No desperdiciar espacio dando créditos al motor de búsqueda, a la empresa de diseño, a la compañía de navegadores favorita o la tecnología tras bambalinas.

G96. Ejercer con moderación al mostrar los premios ganados por el sitio web.

\section{Volver a cargar la página y actualizar}

G97. No actualizar automáticamente la página de inicio para enviar actualizaciones a los usuarios.

G98. Al realizar una actualización, actualizar solo el contenido que realmente haya cambiado, como las actualizaciones de noticias.

\section{Personalización}

G99. Si la página de inicio tiene áreas que brindan información personalizada una vez que se sabe algo sobre el usuario, no brindar una versión genérica del contenido a los usuarios primerizos: crear diferente contenido para ese espacio.

G100. No ofrecer a los usuarios funciones para personalizar el aspecto básico de la interfaz gráfica de la página de inicio, como las combinaciones de colores.

\section{Recopilación de datos del cliente}

G101. No proporcionar enlaces sencillos para registrarse en la página de inicio; en su lugar explicar (o al menos un enlace) los beneficios de registrarse para el cliente.

G102. Explicar a los usuarios los beneficios y la frecuencia de publicación antes de solicitarles su dirección de correo electrónico.

\section{Fechas y horarios}

G105. Mostrar fechas y horas solo para información sensible al tiempo, como noticias, chats en vivo, cotizaciones de la bolsa, y así sucesivamente.

G106. Mostrar a los usuarios la hora en que se actualizó el contenido por última vez, no la hora actual generada por computadora.

G107. Incluir la zona horaria que se está utilizando cada vez que se hace referencia a una hora. 


\subsection{Definición de un sistema porcentual de evaluación para las RSV}

Para llevar a cabo el análisis comparativo de usabilidad de las RSV seleccionadas se ordenaron las guías de las 21 categorías (90 guías en total) identificadas de acuerdo al nivel de importancia que tienen éstas a la hora de analizar la usabilidad de las páginas de inicio. También se asoció a cada categoría un peso porcentual (PP) en relación al 100\%, es decir la suma de los pesos de todas las categorías es igual a 100\%. El peso de las categorías fue fijado de acuerdo al orden de importancia en las que fueron priorizadas. Los pesos porcentuales definidos para cada categoría fueron: Navegación (10\%), Comunicación del propósito del sitio $(6,25 \%)$, Comunicación de información sobre la empresa $(6,25)$, Escritura del contenido (6,25\%), Búsqueda (6.25\%), Gráficos y animación (4,2\%), Diseño gráfico (4,2\%), Publicidad $(4,2 \%)$, URLs $(4,2 \%)$, Enlaces $(4,2 \%)$, Herramientas y atajos de tareas (4\%), Ventanas emergentes y páginas de ensayo (4\%), Volver a cargar la página y actualizar (4\%), Revelación de contenido mediante ejemplos (4\%), Recopilación de datos del cliente (4\%), Títulos de las ventanas (4\%), Fecha y horarios (4\%), Widgets de interfaz de usuario (4\%), Personalización (4\%), Créditos (4\%) y Bienvenidas (4\%). El criterio utilizado para asignar el PP a cada categoría fue asignar el $35 \%$ del peso total a las primeras 5 categorías más importantes asignándole el $10 \%$ a la más importante de todas y el resto a las siguientes 4 , luego el $21 \%$ a las siguientes 5 (4,2\% a cada una), y el restante de $44 \%$ se le asignó a las 11 categorías siguientes. Las 10 primeras categorías que son las más importantes y representativas de acuerdo a priorización que se realizó con anterioridad conforman el $56 \%(35 \%+21 \%)$, mientras que el resto es el $44 \%$.

Por otro lado, para evaluar el nivel de cumplimiento de una RSV con una guía en particular se utilizó una escala porcentual de $0 \%, 50 \%$ y 100\%, la primera representa que no se cumplió con lo propuesto por la guía, la segunda que se cumplió parcialmente y la última que se cumplió en su totalidad. Una vez obtenido el nivel de cumplimiento de las guías pertenecientes a una categoría se realizó la suma de los pesos porcentuales y se los dividió por la cantidad de guías de la categoría para así obtener un promedio de categoría por RSV (PCAT). Para registrar este procedimiento se construyó una tabla con los campos acordes para organizar dicha información (Tabla 2).

Luego de obtener PCAT, se creó una nueva tabla (Tabla 3) para poder representar la relación de los promedios porcentuales obtenido en la (Tabla 2) con el peso que se le había asignado a esa categoría (PP). Se realizó un cálculo para obtener cuanto representaba el PCAT del PP asignado a la categoría: PP * PCAT /100. También se calculó el promedio de cumplimiento por categoría, como así también la diferencia de este con respecto al PP de la categoría. Luego se realizó una sumatoria de todos los pesos porcentuales obtenidos mediante la anterior fórmula, y se pudo obtener el porcentaje de cumplimiento de una RSV (PC-RSV) en relación a todas las guías evaluadas para así poder compararlas.

Por último, se realizó el cálculo de la desviación estándar del conjunto de datos obtenidos luego de aplicar la lista de verificación de usabilidad a las RSV y de realizar la sumatoria de cumplimiento de cada una de las RSV. La desviación estándar (estadístico) de un conjunto de datos de una muestra es una medida que se utiliza para cuantificar la variación o dispersión de un conjunto de datos numéricos ${ }^{5}$. La fórmula para calcular dicha desviación se puede ver en la Figura 1.

\footnotetext{
${ }^{5} \mathrm{https} / / /$ pyme.lavoztx.com/cul-es-la-diferencia-entre-la-muestra-y-la-desviacin-estndar-de-la-poblacin$\underline{5641 . h t m l}$
} 


$$
s=\sqrt{\frac{\sum(x-\bar{x})^{2}}{n-1}}
$$

Figura 1: Fórmula de desviación

Para calcular $s$ se debe realizar la sumatoria de cada dato menos la media aritmética al cuadrado. Es decir: $\left(x_{1}-\bar{x}\right)^{2}+\left(x_{2}-\bar{x}\right)^{2}+\left(x_{3}-\bar{x}\right)^{2}+\cdots+\left(x_{n}-\bar{x}\right)^{2}$. La sumatoria obtenida debe ser dividida por la cantidad de datos obtenidos menos 1 . Y luego calculando la raíz del resultado de la división se obtiene la desviación estándar. En el presente estudio la sumatoria que se realizó tuvo como valor $x$ los valores porcentuales de cumplimiento de cada $\mathrm{RSV}$, y como $\bar{x}$ (media aritmética), el promedio de las mismas. El valor de $n$ es la cantidad de RSV analizadas que en este caso son 5.

Existen tres reglas relacionadas con la desviación estándar que sirven para poder comprender y utilizar el dato calculado: la primera regla es que el $68 \%$ de los valores se ubican a no más de una desviación estándar del promedio (Promedio $+-s$ ) para ambos lados del mismo; la segunda es que el $95 \%$ de los valores se ubican a no más de dos desviaciones estándar del promedio (Promedio +- $(2 * s)$ ) y la tercera es que el $99.7 \%$ de los valores se ubican a no más de tres desviaciones estándar (Promedio +- $(3 * s)$ ).

\section{RESULTADOS}

En la Tabla 2 se encuentran las categorías de las guías de usabilidad, ordenadas de acuerdo a su nivel de importancia de manera ascendente y cada categoría posee los identificados de las guías que pertenecen a esa categoría.

Tabla 2: Evaluación de las guías de usabilidad en cada RSV

\begin{tabular}{|c|c|c|c|c|c|c|}
\hline Categoría & Guía & Youtube & Vimeo & Dailymotion & Metacafe & Break \\
\hline \multirow{6}{*}{ Navegación } & G040 & $100 \%$ & $100 \%$ & $100 \%$ & $100 \%$ & $100 \%$ \\
\hline & G041 & $100 \%$ & $100 \%$ & $100 \%$ & $100 \%$ & $100 \%$ \\
\hline & G042 & $100 \%$ & $100 \%$ & $100 \%$ & $50 \%$ & $100 \%$ \\
\hline & G043 & $0 \%$ & $50 \%$ & $50 \%$ & $0 \%$ & $0 \%$ \\
\hline & G044 & $100 \%$ & $100 \%$ & $100 \%$ & $100 \%$ & $100 \%$ \\
\hline & G046 & $50 \%$ & $100 \%$ & $100 \%$ & $100 \%$ & $50 \%$ \\
\hline $10 \%$ & 6 & $75 \%$ & $92 \%$ & $92 \%$ & $75 \%$ & $75 \%$ \\
\hline \multirow{7}{*}{$\begin{array}{l}\text { Comunicación } \\
\text { del propósito } \\
\text { del sitio }\end{array}$} & G001 & $100 \%$ & $100 \%$ & $100 \%$ & $100 \%$ & $100 \%$ \\
\hline & G002 & $0 \%$ & $0 \%$ & $0 \%$ & $0 \%$ & $0 \%$ \\
\hline & G003 & $0 \%$ & $0 \%$ & $0 \%$ & $0 \%$ & $50 \%$ \\
\hline & G004 & $100 \%$ & $50 \%$ & $0 \%$ & $100 \%$ & $50 \%$ \\
\hline & G005 & $100 \%$ & $100 \%$ & $100 \%$ & $100 \%$ & $100 \%$ \\
\hline & G006 & $100 \%$ & $100 \%$ & $100 \%$ & $100 \%$ & $100 \%$ \\
\hline & G007 & $50 \%$ & $50 \%$ & $50 \%$ & $50 \%$ & $50 \%$ \\
\hline $6.25 \%$ & 7 & $64 \%$ & $57 \%$ & $50 \%$ & $64 \%$ & $64 \%$ \\
\hline \multirow{6}{*}{$\begin{array}{c}\text { Comunicación } \\
\text { de } \\
\text { información } \\
\text { sobre la } \\
\text { empresa }\end{array}$} & G008 & $50 \%$ & $100 \%$ & $100 \%$ & $100 \%$ & $50 \%$ \\
\hline & G009 & $100 \%$ & $100 \%$ & $100 \%$ & $50 \%$ & $0 \%$ \\
\hline & G010 & $100 \%$ & $0 \%$ & $0 \%$ & $0 \%$ & $0 \%$ \\
\hline & G012 & $100 \%$ & $0 \%$ & $0 \%$ & $0 \%$ & $0 \%$ \\
\hline & G013 & $50 \%$ & $0 \%$ & $0 \%$ & $0 \%$ & $0 \%$ \\
\hline & G014 & $100 \%$ & $100 \%$ & $100 \%$ & $100 \%$ & $100 \%$ \\
\hline
\end{tabular}




\begin{tabular}{|c|c|c|c|c|c|c|}
\hline & G015 & $100 \%$ & $50 \%$ & $100 \%$ & $100 \%$ & $100 \%$ \\
\hline & G016 & $50 \%$ & $100 \%$ & $100 \%$ & $0 \%$ & $0 \%$ \\
\hline $6,25 \%$ & 8 & $81 \%$ & $56 \%$ & $62 \%$ & $43 \%$ & $31 \%$ \\
\hline \multirow{11}{*}{$\begin{array}{l}\text { Escritura del } \\
\text { contenido }\end{array}$} & G017 & $100 \%$ & $50 \%$ & $100 \%$ & $100 \%$ & $100 \%$ \\
\hline & G018 & $100 \%$ & $50 \%$ & $100 \%$ & $50 \%$ & $50 \%$ \\
\hline & G019 & $100 \%$ & $50 \%$ & $100 \%$ & $100 \%$ & $100 \%$ \\
\hline & G020 & $100 \%$ & $100 \%$ & $100 \%$ & $100 \%$ & $100 \%$ \\
\hline & G021 & $100 \%$ & $100 \%$ & $100 \%$ & $100 \%$ & $100 \%$ \\
\hline & G022 & $100 \%$ & $100 \%$ & $100 \%$ & $100 \%$ & $100 \%$ \\
\hline & G023 & $100 \%$ & $100 \%$ & $100 \%$ & $100 \%$ & $100 \%$ \\
\hline & G024 & $50 \%$ & $50 \%$ & $50 \%$ & $100 \%$ & $100 \%$ \\
\hline & G026 & $100 \%$ & $100 \%$ & $100 \%$ & $100 \%$ & $100 \%$ \\
\hline & G027 & $100 \%$ & $100 \%$ & $100 \%$ & $100 \%$ & $0 \%$ \\
\hline & G028 & $50 \%$ & $100 \%$ & $100 \%$ & $100 \%$ & $100 \%$ \\
\hline $6,25 \%$ & 11 & $91 \%$ & $82 \%$ & $95 \%$ & $95 \%$ & $86 \%$ \\
\hline \multirow{6}{*}{ Búsqueda } & G047 & $100 \%$ & $100 \%$ & $50 \%$ & $50 \%$ & $100 \%$ \\
\hline & G048 & $100 \%$ & $100 \%$ & $100 \%$ & $100 \%$ & $100 \%$ \\
\hline & G049 & $50 \%$ & $50 \%$ & $50 \%$ & $50 \%$ & $50 \%$ \\
\hline & G050 & $100 \%$ & $100 \%$ & $50 \%$ & $100 \%$ & $50 \%$ \\
\hline & G051 & $100 \%$ & $100 \%$ & $100 \%$ & $100 \%$ & $100 \%$ \\
\hline & G052 & $100 \%$ & $100 \%$ & $100 \%$ & $100 \%$ & $100 \%$ \\
\hline $6,25 \%$ & 6 & $92 \%$ & $92 \%$ & $75 \%$ & $83 \%$ & $83 \%$ \\
\hline \multirow{6}{*}{$\begin{array}{l}\text { Gráficos y } \\
\text { animación }\end{array}$} & G056 & $100 \%$ & $50 \%$ & $100 \%$ & $100 \%$ & $100 \%$ \\
\hline & G057 & $100 \%$ & $100 \%$ & $100 \%$ & $100 \%$ & $100 \%$ \\
\hline & G058 & $100 \%$ & $100 \%$ & $100 \%$ & $100 \%$ & $100 \%$ \\
\hline & G059 & $100 \%$ & $100 \%$ & $100 \%$ & $100 \%$ & $100 \%$ \\
\hline & G060 & $100 \%$ & $100 \%$ & $100 \%$ & $100 \%$ & $100 \%$ \\
\hline & G061 & $100 \%$ & $100 \%$ & $100 \%$ & $100 \%$ & $100 \%$ \\
\hline $4,20 \%$ & 6 & $100 \%$ & $92 \%$ & $100 \%$ & $100 \%$ & $100 \%$ \\
\hline \multirow{6}{*}{ Diseño gráfico } & G063 & $100 \%$ & $100 \%$ & $100 \%$ & $100 \%$ & $50 \%$ \\
\hline & G064 & $100 \%$ & $50 \%$ & $100 \%$ & $100 \%$ & $50 \%$ \\
\hline & G065 & $100 \%$ & $100 \%$ & $100 \%$ & $100 \%$ & $100 \%$ \\
\hline & G066 & $100 \%$ & $100 \%$ & $50 \%$ & $100 \%$ & $100 \%$ \\
\hline & G067 & $100 \%$ & $100 \%$ & $100 \%$ & $100 \%$ & $100 \%$ \\
\hline & G068 & $100 \%$ & $100 \%$ & $100 \%$ & $100 \%$ & $100 \%$ \\
\hline $4,20 \%$ & 6 & $100 \%$ & $92 \%$ & $92 \%$ & $100 \%$ & $83 \%$ \\
\hline Publicidad & G091 & $100 \%$ & $50 \%$ & $100 \%$ & $100 \%$ & $50 \%$ \\
\hline $4,20 \%$ & 1 & $100 \%$ & $50 \%$ & $100 \%$ & $100 \%$ & $50 \%$ \\
\hline \multirow{3}{*}{ URLs } & G077 & $100 \%$ & $50 \%$ & $100 \%$ & $100 \%$ & $100 \%$ \\
\hline & G078 & $100 \%$ & $100 \%$ & $100 \%$ & $100 \%$ & $100 \%$ \\
\hline & G080 & $0 \%$ & $0 \%$ & $0 \%$ & $0 \%$ & $0 \%$ \\
\hline $4,20 \%$ & 3 & $66 \%$ & $50 \%$ & $66 \%$ & $66 \%$ & $66 \%$ \\
\hline \multirow{6}{*}{ Enlaces } & G034 & $100 \%$ & $100 \%$ & $50 \%$ & $100 \%$ & $100 \%$ \\
\hline & G035 & $100 \%$ & $100 \%$ & $100 \%$ & $100 \%$ & $100 \%$ \\
\hline & G036 & $100 \%$ & $100 \%$ & $100 \%$ & $100 \%$ & $100 \%$ \\
\hline & G037 & $0 \%$ & $0 \%$ & $0 \%$ & $50 \%$ & $0 \%$ \\
\hline & G038 & $100 \%$ & $100 \%$ & $100 \%$ & $100 \%$ & $100 \%$ \\
\hline & G039 & $50 \%$ & $50 \%$ & $50 \%$ & $50 \%$ & $50 \%$ \\
\hline $4,20 \%$ & 6 & $75 \%$ & $75 \%$ & $67 \%$ & $83 \%$ & $75 \%$ \\
\hline \multirow{3}{*}{$\begin{array}{c}\text { Herramientas } \\
\text { y atajos de } \\
\text { tareas }\end{array}$} & G053 & $100 \%$ & $50 \%$ & $100 \%$ & $100 \%$ & $100 \%$ \\
\hline & G054 & $100 \%$ & $100 \%$ & $100 \%$ & $100 \%$ & $100 \%$ \\
\hline & G055 & $100 \%$ & $100 \%$ & $100 \%$ & $100 \%$ & $100 \%$ \\
\hline $4 \%$ & 3 & $100 \%$ & $83 \%$ & $100 \%$ & $100 \%$ & $100 \%$ \\
\hline
\end{tabular}




\begin{tabular}{|c|c|c|c|c|c|c|}
\hline \multirow{3}{*}{$\begin{array}{l}\text { Ventanas } \\
\text { emergentes y } \\
\text { páginas de } \\
\text { ensayo }\end{array}$} & G085 & $100 \%$ & $100 \%$ & $100 \%$ & $100 \%$ & $100 \%$ \\
\hline & G086 & $100 \%$ & $100 \%$ & $50 \%$ & $100 \%$ & $100 \%$ \\
\hline & G087 & $100 \%$ & $100 \%$ & $100 \%$ & $100 \%$ & $100 \%$ \\
\hline $4 \%$ & 3 & $100 \%$ & $100 \%$ & $83 \%$ & $100 \%$ & $100 \%$ \\
\hline \multirow{2}{*}{$\begin{array}{l}\text { Volver a } \\
\text { cargar la } \\
\text { página y } \\
\text { actualizar }\end{array}$} & G097 & $100 \%$ & $100 \%$ & $100 \%$ & $100 \%$ & $100 \%$ \\
\hline & G098 & $100 \%$ & $100 \%$ & $100 \%$ & $100 \%$ & $100 \%$ \\
\hline $4 \%$ & 2 & $100 \%$ & $100 \%$ & $100 \%$ & $100 \%$ & $100 \%$ \\
\hline \multirow{4}{*}{$\begin{array}{l}\text { Revelación de } \\
\text { contenido } \\
\text { mediante } \\
\text { ejemplos }\end{array}$} & G029 & $100 \%$ & $100 \%$ & $100 \%$ & $100 \%$ & $100 \%$ \\
\hline & G030 & $100 \%$ & $100 \%$ & $100 \%$ & $100 \%$ & $100 \%$ \\
\hline & G031 & $100 \%$ & $100 \%$ & $100 \%$ & $50 \%$ & $50 \%$ \\
\hline & G032 & $100 \%$ & $50 \%$ & $50 \%$ & $0 \%$ & $50 \%$ \\
\hline $4 \%$ & 4 & $100 \%$ & $88 \%$ & $66 \%$ & $63 \%$ & $75 \%$ \\
\hline \multirow{2}{*}{$\begin{array}{l}\text { Recopilación } \\
\text { de datos del } \\
\text { cliente }\end{array}$} & G101 & $100 \%$ & $0 \%$ & $0 \%$ & $0 \%$ & $0 \%$ \\
\hline & G102 & $50 \%$ & $0 \%$ & $50 \%$ & $50 \%$ & $50 \%$ \\
\hline $4 \%$ & 2 & $75 \%$ & $0 \%$ & $25 \%$ & $25 \%$ & $25 \%$ \\
\hline \multirow{5}{*}{$\begin{array}{l}\text { Títulos de las } \\
\text { ventanas }\end{array}$} & G072 & $100 \%$ & $100 \%$ & $100 \%$ & $100 \%$ & $100 \%$ \\
\hline & G073 & $100 \%$ & $100 \%$ & $100 \%$ & $100 \%$ & $0 \%$ \\
\hline & G074 & $100 \%$ & $100 \%$ & $100 \%$ & $100 \%$ & $100 \%$ \\
\hline & G075 & $0 \%$ & $100 \%$ & $100 \%$ & $100 \%$ & $100 \%$ \\
\hline & G076 & $100 \%$ & $0 \%$ & $100 \%$ & $0 \%$ & $0 \%$ \\
\hline $4 \%$ & 5 & $80 \%$ & $80 \%$ & $100 \%$ & $80 \%$ & $60 \%$ \\
\hline \multirow{3}{*}{$\begin{array}{l}\text { Fechas y } \\
\text { horarios }\end{array}$} & G105 & $100 \%$ & $0 \%$ & $0 \%$ & $100 \%$ & $100 \%$ \\
\hline & G106 & $50 \%$ & $0 \%$ & $0 \%$ & $50 \%$ & $50 \%$ \\
\hline & G107 & $0 \%$ & $0 \%$ & $0 \%$ & $0 \%$ & $0 \%$ \\
\hline $4 \%$ & 3 & $50 \%$ & $0 \%$ & $0 \%$ & $50 \%$ & $50 \%$ \\
\hline \multirow{3}{*}{$\begin{array}{l}\text { Widgets de } \\
\text { interfaz de } \\
\text { usuario }\end{array}$} & G069 & $100 \%$ & $100 \%$ & $100 \%$ & $100 \%$ & $100 \%$ \\
\hline & G070 & $100 \%$ & $100 \%$ & $100 \%$ & $100 \%$ & $50 \%$ \\
\hline & G071 & $50 \%$ & $100 \%$ & $100 \%$ & $100 \%$ & $50 \%$ \\
\hline $4 \%$ & 3 & $83 \%$ & $100 \%$ & $100 \%$ & $100 \%$ & $66 \%$ \\
\hline \multirow{2}{*}{$\begin{array}{c}\text { Personalizació } \\
\mathrm{n}\end{array}$} & G099 & $50 \%$ & $50 \%$ & $50 \%$ & $50 \%$ & $50 \%$ \\
\hline & G100 & $0 \%$ & $100 \%$ & $100 \%$ & $100 \%$ & $100 \%$ \\
\hline $4 \%$ & 2 & $25 \%$ & $75 \%$ & $75 \%$ & $75 \%$ & $75 \%$ \\
\hline \multirow{2}{*}{ Créditos } & G095 & $100 \%$ & $50 \%$ & $100 \%$ & $100 \%$ & $100 \%$ \\
\hline & G096 & $100 \%$ & $100 \%$ & $100 \%$ & $100 \%$ & $100 \%$ \\
\hline $4 \%$ & 2 & $100 \%$ & $75 \%$ & $100 \%$ & $100 \%$ & $100 \%$ \\
\hline Bienvenidas & G092 & $100 \%$ & $100 \%$ & $100 \%$ & $100 \%$ & $100 \%$ \\
\hline $4 \%$ & 1 & $100 \%$ & $100 \%$ & $100 \%$ & $100 \%$ & $100 \%$ \\
\hline
\end{tabular}

En la Tabla 3 se muestran las categorías, las RSV y el porcentaje de cumplimiento de las guías de una categoría. También se puede apreciar el promedio de cumplimiento por categoría, como así también la diferencia entre el promedio de la categoría respecto del PP de la categoría. También se utilizaron los medios de representación de gráficos de barra y de columnas para presentar la información obtenida de una manera más sencilla y clara. Se utilizó la gráfica de columnas (Figura 2) para mostrar el porcentaje de cumplimiento de usabilidad de páginas de inicio que fue aplicada a cada RSV, es decir el PC-RSV, respecto del $100 \%$ ideal y esperado. El gráfico de barras (Figura 3) muestra la diferencia en porcentaje que tiene cada categoría con respecto del PP ideal y esperado para la misma. 
Por ejemplo, en la categoría Navegación de Youtube el porcentaje de cumplimiento es de $7,50 \%$ del $10 \%$ que representa esa categoría. Además, la categoría es cumplida en promedio por un $8,18 \%$, y este promedio tiene una diferencia del $1,82 \%$ respecto del PP (10\%).

Tabla 3: Resumen por categoría de porcentaje de evaluación de las guías en las RSV

\begin{tabular}{|c|c|c|c|c|c|c|c|c|}
\hline Categoría & $\mathbf{P P}$ & Youtube & Vimeo & Dailymotion & Metacafe & Break & Promedio & Diferencia \\
\hline Navegación & $10 \%$ & $7,50 \%$ & $9,20 \%$ & $9,20 \%$ & $7,50 \%$ & $7,50 \%$ & $8,18 \%$ & $1,82 \%$ \\
\hline $\begin{array}{l}\text { Comunicación del } \\
\text { propósito del sitio }\end{array}$ & $6,25 \%$ & $4 \%$ & $3,56 \%$ & $3,12 \%$ & $4 \%$ & $4 \%$ & $3,74 \%$ & $2,51 \%$ \\
\hline $\begin{array}{c}\text { Comunicación de } \\
\text { información sobre } \\
\text { la } \\
\text { empresa }\end{array}$ & $6,25 \%$ & $5,06 \%$ & $3,50 \%$ & $3,87 \%$ & $2,69 \%$ & $1,94 \%$ & $3,41 \%$ & $2,84 \%$ \\
\hline $\begin{array}{l}\text { Escritura del } \\
\text { contenido }\end{array}$ & $6,25 \%$ & $5,69 \%$ & $5,12 \%$ & $5,94 \%$ & $5,94 \%$ & $5,37 \%$ & $5,61 \%$ & $0,64 \%$ \\
\hline Búsqueda & $6,25 \%$ & $5,75 \%$ & $5,75 \%$ & $4,69 \%$ & $5,19 \%$ & $5,19 \%$ & $5,31 \%$ & $0,94 \%$ \\
\hline $\begin{array}{l}\text { Gráficos y } \\
\text { animación }\end{array}$ & $4,20 \%$ & $4,20 \%$ & $3,86 \%$ & $4,20 \%$ & $4,20 \%$ & $4,20 \%$ & $4,13 \%$ & $0,07 \%$ \\
\hline Diseño gráfico & $4,20 \%$ & $4,20 \%$ & $3,86 \%$ & $3,86 \%$ & $4,20 \%$ & $3,49 \%$ & $3,92 \%$ & $0,28 \%$ \\
\hline Publicidad & $4,20 \%$ & $4,20 \%$ & $2,10 \%$ & $4,20 \%$ & $4,20 \%$ & $2,10 \%$ & $3,36 \%$ & $0,84 \%$ \\
\hline URLs & $4,20 \%$ & $2,77 \%$ & $2,10 \%$ & $2,77 \%$ & $2,77 \%$ & $2,77 \%$ & $2,64 \%$ & $1,56 \%$ \\
\hline Enlaces & $4,20 \%$ & $3,15 \%$ & $3,15 \%$ & $2,81 \%$ & $3,49 \%$ & $3,15 \%$ & $3,15 \%$ & $1,05 \%$ \\
\hline $\begin{array}{c}\text { Herramientas y } \\
\text { atajos de } \\
\text { tareas }\end{array}$ & $4 \%$ & $4 \%$ & $3,32 \%$ & $4 \%$ & $4 \%$ & $4 \%$ & $3,86 \%$ & $0,14 \%$ \\
\hline $\begin{array}{c}\text { Ventanas } \\
\text { emergentes y } \\
\text { páginas de ensayo } \\
\end{array}$ & $4 \%$ & $4 \%$ & $4 \%$ & $3,32 \%$ & $4 \%$ & $4 \%$ & $3,86 \%$ & $0,14 \%$ \\
\hline $\begin{array}{c}\text { Volver a cargar la } \\
\text { página y } \\
\text { actualizar }\end{array}$ & $4 \%$ & $4 \%$ & $4 \%$ & $4 \%$ & $4 \%$ & $4 \%$ & $4,00 \%$ & $0,00 \%$ \\
\hline $\begin{array}{l}\text { Revelación de } \\
\text { contenido } \\
\text { mediante } \\
\text { ejemplos } \\
\end{array}$ & $4 \%$ & $4 \%$ & $3,52 \%$ & $3,52 \%$ & $2,52 \%$ & $3 \%$ & $3,31 \%$ & $0,69 \%$ \\
\hline $\begin{array}{c}\text { Recopilación de } \\
\text { datos } \\
\text { del cliente } \\
\end{array}$ & $4 \%$ & $3 \%$ & $0 \%$ & $1 \%$ & $1 \%$ & $1 \%$ & $1,20 \%$ & $2,80 \%$ \\
\hline $\begin{array}{c}\text { Títulos de las } \\
\text { ventanas }\end{array}$ & $4 \%$ & $3,20 \%$ & $3,20 \%$ & $4 \%$ & $3,20 \%$ & $2,4 \%$ & $3,20 \%$ & $0,80 \%$ \\
\hline Fechas y horarios & $4 \%$ & $2 \%$ & $0 \%$ & $0 \%$ & $2 \%$ & $2 \%$ & $1,20 \%$ & $2,80 \%$ \\
\hline $\begin{array}{c}\text { Widgets de } \\
\text { interfaz de } \\
\text { usuario } \\
\end{array}$ & $4 \%$ & $3,32 \%$ & $4 \%$ & $4 \%$ & $4 \%$ & $2,64 \%$ & $3,59 \%$ & $0,41 \%$ \\
\hline Personalización & $4 \%$ & $1 \%$ & $3 \%$ & $3 \%$ & $3 \%$ & $3 \%$ & $2,60 \%$ & $1,40 \%$ \\
\hline Créditos & $4 \%$ & $4 \%$ & $3 \%$ & $4 \%$ & $4 \%$ & $4 \%$ & $3,80 \%$ & $0,20 \%$ \\
\hline Bienvenidas & $4 \%$ & $4 \%$ & $4 \%$ & $4 \%$ & $4 \%$ & $4 \%$ & $4,00 \%$ & $0,00 \%$ \\
\hline PC-RSV & $100 \%$ & $83,04 \%$ & $74,24 \%$ & $\mathbf{7 9 , 5 0 \%}$ & $79,90 \%$ & $73,75 \%$ & & \\
\hline
\end{tabular}




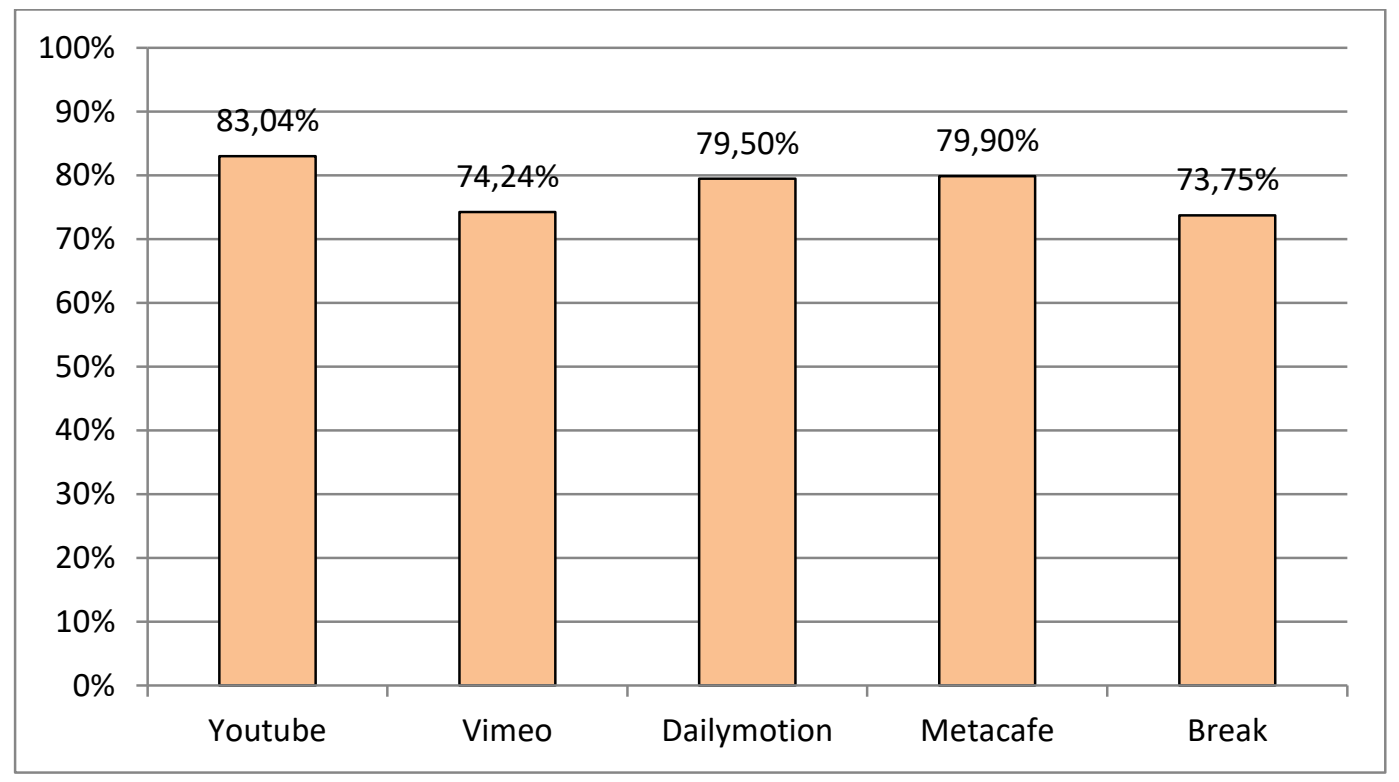

Figura 2: Distribución de los porcentajes de cumplimento de usabilidad

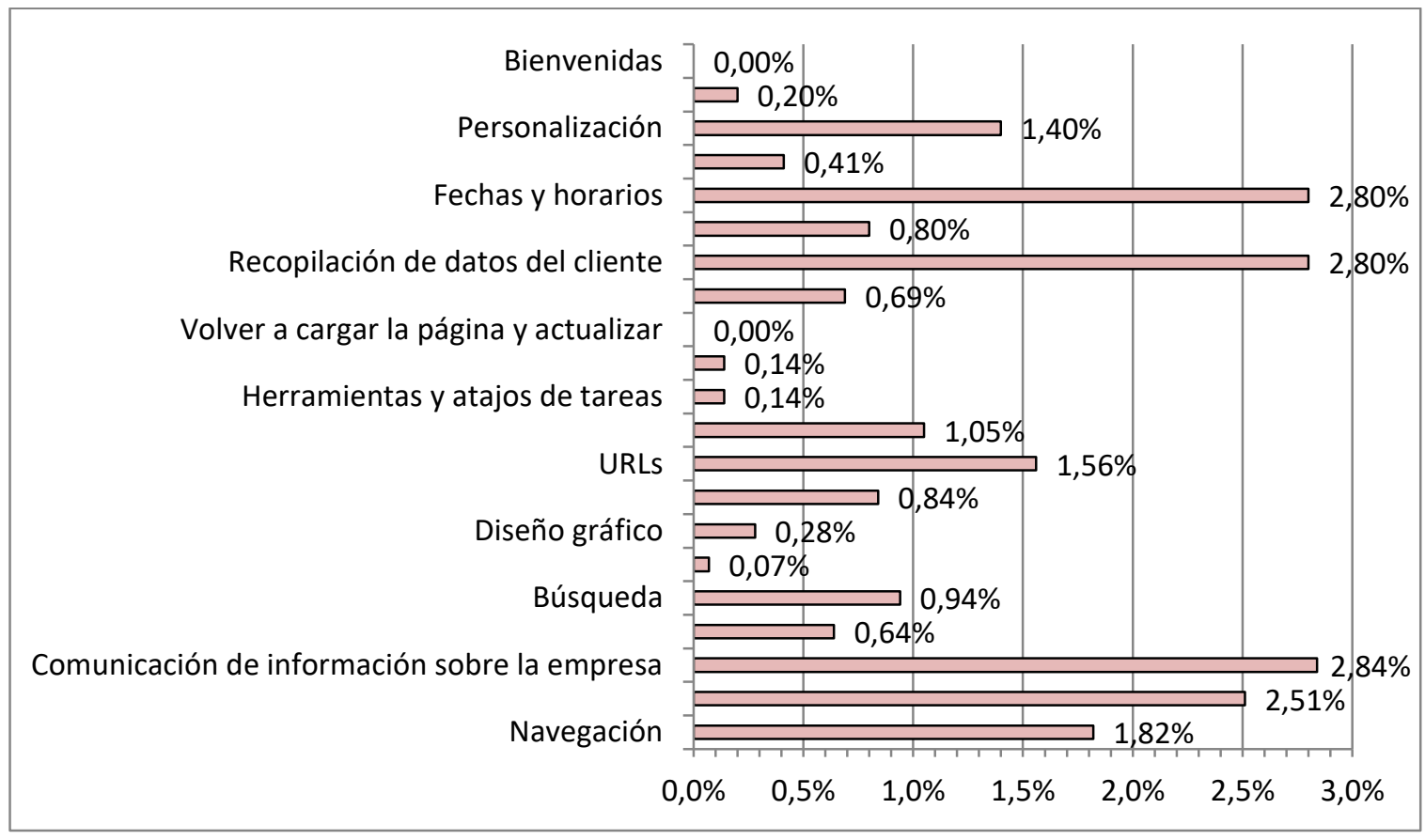

Figura 3: Distribución de las diferencias porcentuales con respecto a PP de cumplimiento de usabilidad por categoría

Además de las tablas presentadas, fue necesario realizar el cálculo de la desviación estándar, pues esta medida de dispersión indica qué tan alejados están los datos con respecto al valor promedio. A partir de este breve análisis fue posible afirmar si alguno de los sitios presentaba mucha diferencia con respecto a los demás, ya sea por su baja o alta calificación global. Los pasos realizados para realizar el cálculo de la desviación estándar fueron los siguientes:

I. Definición del conjunto de datos

El conjunto de datos son los valores porcentuales totales obtenidos en la Tabla 3. Conjunto de datos $=\{83,04 ; 74,24 ; 79,50 ; 79,90 ; 73,75\}$ y $n=5$. 
II. Cálculo del promedio del conjunto de datos

$\bar{x}=(83,04+74,24+79,50+79,90+73,75) \div 5$

$\bar{x} \approx 78,09$

III. Sumatoria de los cuadrados de la distancia entre cada dato y el promedio

$$
\begin{gathered}
\sum(x-\bar{x})^{2}=(83,04-78,09)^{2}+(74,24-78,09)^{2}+(79,50-78,09)^{2}+\ldots \\
\ldots(79,90-78,09)^{2}+(73,75-78,09)^{2} \\
\sum(x-\bar{x})^{2}=24,50+14,82+1,99+3,28+18,84 \\
\sum(x-\bar{x})^{2}=63,43
\end{gathered}
$$

IV. Reemplazando los valores en la fórmula para obtener la desviación estándar

$$
s=\sqrt{\frac{63,43}{5-1}} \rightarrow s=\sqrt{\frac{63,43}{4}} \rightarrow s=\sqrt{15,86} \rightarrow s=3,98
$$

La desviación estándar del conjunto de datos $\{83,04 ; 74,24 ; 79,50 ; 79,90 ; 73,75\}$ es de 3,98. Aplicando las reglas de la desviación estándar que fueron explicadas en la sección anterior se puede obtener que el $68 \%$ de los valores se ubican a no más de una desviación estándar del promedio $(78,09 \%+-3,98)$; el $95 \%$ de los valores se ubican a no más de dos desviaciones estándar del promedio (es decir, 78,09\%+-(2*3,98)); y el $99.7 \%$ de los valores se ubican a no más de tres desviaciones estándar (es decir, 78,09\%+-(3*3,98)).

\section{CONCLUSIONES}

Las redes sociales de videos analizadas presentan diferentes grados de cumplimiento respecto a las guías de usabilidad de páginas de inicio que se aplicaron. Si bien algunas RSV logran cumplir por completo con guías de alguna categoría específica, ninguna logra cumplir con todas las categorías por completo. En general todas las RSV obtuvieron un PC-RSV mayor al $70 \%$, lo cual significa que los niveles de usabilidad en las páginas de inicio de las RSV son altos. A continuación, se detalla el grado de cumplimiento de las RSV en orden ascendente: Break (73,75\%), Vimeo (74,24\%), Dailymotion $(79,50 \%)$, Metacafe $(79,90)$ y Youtube $(83,04 \%)$. Se puede distinguir que solamente Break y Vimeo tienen PC-RSV ubicados por debajo del 75\%, el resto de las RSV superan este valor y la RSV que tiene el PC-RSV más alto es Youtube. Los PC-RSV tienen una desviación estándar de 3,98 lo cual significa que los resultados obtenidos tienen un grado de dispersión reducido entre ellos.

Dado que las reglas relacionadas con la desviación estándar definen que aproximadamente el $68 \%$ de los valores se ubican a no más de una desviación estándar del promedio $(78,09 \%+-$ $3,98)$, que el $95 \%$ de los valores se ubican a no más de dos desviaciones estándar del promedio (es decir, $78,09+-(2 * 3,98)$ ) y que el $99.7 \%$ de los valores se ubican a no más de tres 
desviaciones estándar (es decir, 78,09+-(3*3,98)). Entonces se puede afirmar que la usabilidad de las páginas de inicio web de RSV se podría encontrar en un rango desde $66,15 \%$ hasta $90,03 \%$. Esta regla, también permite asegurar que no existe una superioridad notoria de Youtube con respecto al promedio ni tampoco una tan baja calidad de Break al punto de considerarse una RSV con poca usabilidad.

Considerando las 5 categorías con los PP más altos, en Navegación (10\%) las RSV que obtuvieron un PCAT más alto son Vimeo y Dailymotion con el 9,20\%, mientras que Youtube, Break y Metacafe lo cumplieron en menor medida con un PCAT de 7,50\%, la navegación es cumplida satisfactoriamente por las RSV en un intervalo de 7,50\% y 9,20\%. En la categoría de Comunicación del propósito del sitio (6.25\%) se obtuvieron PCAT relativamente bajos lo cual implica que es un aspecto del diseño de la página de inicio desatendido, los más altos son los de Youtube, Metacafe y Break con un PCAT de 4\%, mientras Dailymotion con el 3,12 es el más bajo. La categoría de Comunicación de información sobre la empresa $(6.25 \%)$ tiene como única RSV con mayor PCAT a Youtube con el 5,06\%. y en contraste Break con el PCAT más bajo de $1,94 \%$, en esta categoría los PCAT obtenidos se encuentran en un rango bastante amplio 1,94\% - 5,06 lo cual implica que es variable la manera de comunicar la información sobre la empresa en las RSV. En cuanto a la categoría de Escritura de contenido (6.25\%), la mayoría de las RSV cumplieron con más del 5\%, pero Dailymotion y Metacafe se destacan con el PCAT más alto que es $5,94 \%$, y por otro lado Vimeo con el 5,12\% tiene el PCAT más bajo, las RSV cumplen con la categoría en un intervalo de 5,12\% a 5,94\% muy cercano al total, lo cual implica un alto cumplimiento con las guías relacionadas con la escritura. Y por último en la categoría de Búsqueda $(6.25 \%)$ las RSV que obtuvieron PCAT más altos fueron Youtube y Vimeo con el 5,75, mientras que Dailymotion con el 4,69 obtuvo el PCAT más bajo en la categoría. La búsqueda en las RSV al igual que la escritura posee un alto nivel de cumplimiento.

Se puede destacar que Youtube es la única RSV en tener el mejor PCAT en 3 (Comunicación del propósito del sitio, Comunicación de información sobre la empresa y Búsqueda) de las 5 categorías con PP más alto y solo se encuentra con el PCAT más bajo en la categoría de Navegación, aspecto que no es una fortaleza en esta RSV. También cabe señalar que en la categoría Navegación, la cual tiene el mayor PP, las RSV que más se acercan a cumplir con el $100 \%$ del PP son Vimeo y Dailymotion. Además de las 5 categorías más importantes, las que fueron cumplidas con mayor cercanía al PP son las de Escritura del contenido y Búsqueda en las 5 RSV analizadas.

Existen categorías que tienen un nivel de cumplimiento promedio que no supera el 0,5 de diferencia respecto del PP ideal esperado. En otras palabras, todas las RSV cumplen casi al 100\% con las guías de usabilidad de las categorías de Gráficos y animación; Diseño gráfico; Herramientas y atajos de tareas; Ventanas emergentes y páginas de ensayo; Volver a cargar la página y actualizar; Widgets de interfaz de usuario; Créditos y Bienvenidas. Por otro lado, existen categorías que tienen un nivel de cumplimiento promedio que superan el 2,0 de diferencia respecto al PP ideal esperado. Es decir, que las RSV deberían mejorar en el cumplimiento de las categorías Comunicación del propósito del sitio; Comunicación de información sobre la empresa; Recopilación de datos del cliente; Fecha y horarios. De las categorías nombradas la Comunicación del propósito del sitio y Comunicación de información sobre la empresa son las que poseen mayor PP después de la categoría Navegación, los diseñadores de RSV y también las RSV analizadas deberían prestar atención a la mejora de estos aspectos de sus páginas de inicio. 
Por otro lado, considerando las 5 categorías con los PP más bajos, que si bien no son tan importantes o no son una gran influencia para definir el nivel de usabilidad de una página de inicio, es interesante hacer un breve análisis de los porcentajes de cumplimiento para poder detectar falencias a mejorar que tendrán que ser tenidas en cuenta en segundo lugar o con menor prioridad que las detectadas en categorías con mayor PP. Entre estas categorías se encuentran Fechas y horarios (4\%), en esta categoría Youtube, Metacafe y Break tienen un PCAT de $2 \%$, mientras que Vimeo y Dailymotion no cumplen con esta categoría, esto significa que el uso de Fechas y horarios no está siendo presentado adecuadamente o el recurso no está siendo utilizado de manera que ayude al usuario al usar la página de inicio. En la categoría Widgets de interfaz de usuario (4\%) las RSV Vimeo, Dailymotion y Metacafe cumplen completamente con las guías de esta categoria correspondiente a un PCAT de 4\%, por otro lado Break con el 2,64\% es el PCAT más bajo, esto demuestra que 4 de las 5 RSV cumplen con excelencia las guías de usabilidad sugeridas en esta categoría. En la categoría de Personalización (4\%), Vimeo, Dailymotion, Metacafe y Break tienen un PCAT de 3\%, mientras que Youtube uno de $1 \%$, esto permite afirmar que Youtube debe revisar este aspecto del diseño para mejorar la usabilidad de su página de inicio. La categoría de Créditos (4\%) tiene a Youtube, Dailymotion, Metacafe y Break cumpliendo con el 4\%, mientras que Vimeo solo con el $3 \%$, en esta categoría se puede ver un alto cumplimiento de las guías propuestas. Finalizando, en la categoría de Bienvenidas (4\%) todas las RSV obtuvieron un PCAT de 4\%, aspecto a destacar de todas las RSV.

Luego de todo el análisis de la información obtenida se puede concluir que todas las RSV analizadas en este trabajo presentan altos niveles de cumplimiento de aspectos del diseño de su página de inicio que ayuda a poseer un nivel de usabilidad altamente aceptable. Además, en términos generales también se puede afirmar, que a través del estudio de esta muestra de 5 RSV y de un previo análisis estadístico, todas las RSV existentes al aplicarle la lista de verificación de usabilidad confeccionada tendrán un porcentaje de cumplimiento alto y mayor al 50\% que se encontrará en un rango de $66,15 \%$ - 90,03\%.

Desde luego todo el análisis realizado en las RSV puede replicarse y aplicarse a otro tipo de sitios web. Para así obtener información similar que destaque debilidades y fortalezas del sitio o la página analizados.

\section{REFERENCIAS}

KITCHENHAM B. (1996). DESMET: A method for evaluating Software Engineering methods and tools. Department of Computer Science University of Keele. Keele, Staffordshire, UK. Technical Report TR96-09.

KRUG S. (2006). No me hagas pensar: Una aproximación a la usabilidad en la Web. 2da edición. Editorial: Pearson Prentice Hall. ISBN: 84-8322-286-8

LEAVITT M. O. y SHNEIDERMAN, B. (2004). Research-Based Web Design \& Usability Guidelines. Washington, U.S.: U.S. Government Printing Office. ISBN 0-16-0762707

MATOS MAREÑO, R. (2013). La usabilidad como factor de calidad de páginas web. Especialización en Ingeniería de Software, Facultad de Informática, Universidad Nacional de La Plata (UNLP).

NIELSEN J. (2000). Designing Web Usability. 1ra Edición. Editorial: New riders. ISBN: 978-156-20-5810-4 
NIELSEN J. (2001). 113 Design Guidelines for Homepage Usability. Nielsen Norman Group. Disponible en: https://www.nngroup.com/articles/113-design-guidelines-homepageusability/ (Último acceso: 18/12/219)

NIELSEN J. (2012). Usability 101: Introduction to Usability. Nielsen Norman Group. Disponible en: https://www.nngroup.com/articles/usability-101-introduction-tousability/ (Último acceso: 18/12/219)

PERURENA CANCIO L., MORÁGUEZ BERGUES M. (2013). Usabilidad de los sitios Web, los métodos y las técnicas para la evaluación. Revista Cubana de Información en Ciencias de la Salud. 2013;24(2):176-194. Universidad de La Habana (Cuba).

PRESSMAN R. (2010). Ingeniería del software, Un enfoque práctico. 7ma edición. Editorial: Mc Graw Hill. ISBN: 978-607-15-0314-5

SANCHEZ W. (2011). La usabilidad en Ingeniería de Software: definición y características. Revista de Ingeniería e Innovación de la Facultad de Ingeniería, Universidad Don Bosco, Año 1, No. 2. pp. 7-21. ISSN: 2221-1136.

SERRANO MASCARAQUE E. (2009). Accesibilidad vs usabilidad web: evaluación y correlación. Universidad de Alcalá (Madrid), España. https://doi.org/10.22201/iibi.0187358xp.2009.48.16970 


\begin{abstract}
ANEXO 1

\section{Comunicación de información sobre la empresa}

G11. Presentar una imagen unificada al cliente, en la que el sitio web es uno de los puntos de contacto en lugar de una entidad por sí misma. No separar la presencia web del resto de la empresa refiriéndose a "empresa.com" como diferente de "Empresa".
\end{abstract}

\title{
Escritura del contenido
}

G25. Deletrear las abreviaturas, los inicialismos y los acrónimos, y seguir inmediatamente por la abreviatura, en la primera instancia.

\section{Archivos y accesos a contenido del pasado}

G33. Facilitar el acceso a cualquier elemento que haya aparecido recientemente en la página de inicio, por ejemplo, en las últimas dos semanas o meses, proporcionando una lista de las características recientes, así como poner los elementos recientes en los archivos permanentes.

\section{Navegación}

G45. Si se tiene una función de carrito de compras en el sitio, incluir un enlace en la página de inicio.

\section{Gráficos y animación}

G62. Permitir que los usuarios elijan si desean ver una introducción animada del sitio.

\section{URLs}

G79. Si se encuentra disponible, registrar nombres de dominio para deletreos, abreviaturas o errores ortográficos comunes.

\section{Noticias y comunicados}

G81. Los titulares deben ser sucintos, pero descriptivos, para brindar la máxima información en tan pocas palabras como posible.

G82. Escribir y editar resúmenes específicos para comunicados de prensa e historias de noticias que se presenten en la página principal.

G83. Enlazar los titulares, en lugar de la cubierta, a la noticia completa.

G84. Siempre que todas las noticias en la página de inicio se produzcan dentro de la semana, no hay necesidad de enumerar la fecha y la hora en la cubierta de cada historia, a menos que sea realmente una noticia de última hora que tiene frecuentes actualizaciones.

\section{Publicidad}

G88. Mantener anuncios para empresas externas en la periferia de la página.

G89. Mantener los anuncios externos (anuncios de compañías que no sean del sitio) tan pequeños y discretos como sea posible al contenido de la página de inicio. 
G90. Si se colocan anuncios fuera del área de banner estándar en la parte superior de la página, etiquetarlos como publicidad para que los usuarios no los confundan con el contenido del sitio.

\section{Comunicación de problemas técnicos y manejo de emergencias}

G93. Mostrar claramente en la página de inicio si el sitio web está fuera de servicio o si algunas partes importantes del sitio web no están operativas.

G94. Tener un plan para manejar contenido crítico en el sitio web en caso de una emergencia.

\section{Fomento de la comunidad}

G103. Si se admiten comunidades de usuarios con chat u otras funciones de discusión, no mostrar enlaces genéricos a ellos. En su lugar, hacer una lista de los temas de discusión reales y proporcionar los horarios en la página de inicio.

G104. No ofrecer un registro de "Libro de visitas" para sitios de negocios.

\section{Fechas y horarios}

G108. Usar abreviaturas estándar, como pm o PM. No abreviar como "p".

G109. Deletrear el mes, o usar abreviaturas de mes, no números.

\section{Cotizaciones de acciones y números de visualización}

G110. Indicar el porcentaje de cambio, no solo los puntos ganados o perdidos en las cotizaciones de acciones.

G111. Escribir las abreviaturas de las acciones a menos que la abreviatura sea completamente clara, como "IBM".

G112. Usar un separador de miles apropiado para la localidad pertinente para números que tengan cinco o más dígitos.

G113. Alinear puntos decimales al mostrar columnas de números. 